\title{
EXO70A2 is critical for the exocyst complex function in Arabidopsis pollen
}

Vedrana Marković ${ }^{1,2}$, Fatima Cvrčková1, Martin Potocký², PřemysI Pejchar², Eva Kollárová1, Ivan Kulich ${ }^{1}$, Lukáš Synek², Viktor Žárský1,2

${ }^{1}$ Department of Experimental Plant Biology, Faculty of Science, Charles University, Vinicna 5, 12844 Prague 2, Czech Republic

2 Institute of Experimental Botany, Czech Academy of Sciences, Rozvojova 263, 16502 Prague 6, Czech Republic

Authors' Contributions:

V.M., L.S., I.K., V.Z. planned and designed the research. V.M., L.S. P.P., E.K. performed biological experiments. F.C., M.P. conducted the phylogenetic analysis. V.M., L.S., F.C., V.Z. wrote the manuscript.

Corresponding authors

Viktor Žárský - viktor.zarsky@natur.cuni.cz

Lukáš Synek - synek@ueb.cas.cz

\begin{abstract}
Pollen development, pollen grain germination and pollen tube elongation are crucial biological processes in angiosperm plants that need precise regulation to deliver sperm cells to fertilize ovules. Pollen grains undergo two major developmental switches: dehydration characterized by metabolic quiescent state, and rehydration upon pollination that leads to extraordinary metabolic and membrane trafficking activity, resulting in germination and rapid tip growth of pollen tubes. To sustain these processes, many plant housekeeping genes evolved their pollen-specific paralogs. Highly polarized secretion at a growing pollen tube tip requires the exocyst tethering complex responsible for specific targeting of secretory vesicles to the plasma membrane. Here, we describe that EXO70A2 (At5g52340) is the main exocyst EXO70 isoform in Arabidopsis pollen, which governs the conventional secretory function of the exocyst, analogically to EXO70A1 (At5g03540) in the sporophyte. Our analysis of a CRISPR-generated exo70a2 mutant revealed that EXO70A2 is essential for efficient pollen maturation, pollen grain germination and pollen tube growth. GFP:EXO70A2 was localized similarly to other exocyst subunits to the apical domain in growing pollen tube tips characterized by intensive exocytosis. Moreover, EXO70A2 could substitute for the EXO70A1 function in the sporophyte, indicating functional redundancy of these two closely related isoforms. Phylogenetic analysis revealed that the ancient duplication of EXO70A to two (or more) paralogs, one of which is highly expressed in pollen, occurred independently in monocots and dicots. In summary, EXO70A2 is a crucial component of the exocyst complex in the Arabidopsis pollen required for efficient plant sexual reproduction.
\end{abstract}

\section{KEY WORDS}

exocyst, EXO70A2, EXO70A1, pollen development, pollen tube, tip growth, polarized secretion

\section{ABBREVIATIONS}

CDS - coding sequence

GFP - green fluorescent protein 
NBT - nitroblue tetrazolium

PM - plasma membrane

PME - pectin methylesterases

ROS - reactive oxygen species

WT - wild type

\section{INTRODUCTION}

The pollen tube in Angiosperms represents an extremely elongated cellular structure, that emerges from a rehydrated pollen grain upon its landing on a stigma and delivers two sperm cells to fertilize an ovule. To reach the ovule, the pollen tube navigates through pistil tissues by highly polarized tip growth, restricted to the pollen tube apex. In this tightly regulated process, cell polarity maintenance mechanisms, addition of new membrane material and secretion of cell wall components are essential. Intensive exocytosis is localized to the very tip, followed by a subapical domain where endocytosis takes place to recycle the excess of membranes delivered in secretory vesicles (for review, see e.g. Hepler and Winship, 2015). Other key factors essential for the pollen tube tip growth include small GTPases and a $\mathrm{Ca}^{2+}$-signaling network regulating actin cytoskeleton dynamics (for review, see e.g. Cai et al., 2015). The cell wall of pollen tubes has a unique structure consisting of two layers: a pectinaceous and cellulose layer secreted at the apex, and an additional callose layer deposited in more distant regions from the tip. The spatial distribution of the cell wall components plays a critical role in the pollen tube morphogenesis (Chebli et al., 2012). Because pollen tubes are a simplified cell-autonomous system and exhibit highly polarized growth, they render an excellent model in cell biology for studies of cell polarity and regulation of secretion (e.g. Chebli et al., 2013; Qin and Dong, 2015). Precisely regulated membrane trafficking is crucial not only for pollen tube growth, but also for a previous stages of pollen development - pollen grain maturation and germination (Kang et al., 2003; Peng et al., 2011; Paul et al., 2016).

One of the fundamental regulators of polarized secretion is the exocyst - an evolutionarily conserved protein complex discovered due to its role in docking and tethering of secretory vesicles to specific sites at the plasma membrane (PM), facilitating subsequent fusion of secretory vesicles with the target membrane mediated by the SNARE proteins. The exocyst, first described in yeasts and mammals, consists of eight subunits - Sec3, Sec5, Sec6, Sec8, Sec10, Sec15, Exo70, and Exo84 (TerBush et al., 1996; Guo et al., 1999), with Sec3 and Exo70 generally believed to be responsible for targeting the complex to the PM, while the other subunits form the core of the complex (Boyd et al., 2004; He et al., 2007; Pleskot et al., 2015). Plant genomes encode all exocyst subunits (Cvrčková et al., 2001; Eliáš et al. 2003) that form a functional complex (Hála et al., 2008; Fendrych et al., 2010) and engage in cellular processes requiring targeted secretion, including root hair and pollen tube elongation (Cole et al., 2005; Synek et al., 2006; Hála et al., 2008; Synek et al., 2017), cytokinesis (Fendrych et al., 2010; Rybak et al., 2014), secondary cell wall deposition in trichomes and during xylem development (Kulich et al., 2015; Kubátová et al., 2019; Vukašinović et al., 2017), localized deposition of seed coat pectins (Kulich et al., 2010), transport of PIN auxin carriers to the PM (Drdová et al., 2013), and response to pathogens (Pečenková et al., 2011; Sabol et al., 2017). Viable mutants of $A$. thaliana defective in exocyst subunits (sec8-4, sec15b, exo70a1, exo84b) exhibit dwarfish growth with pleiotropic developmental defects (Cole et al., 2005; Synek et al., 2006; Hála et al., 2008; Fendrych et al., 2010; Batystová et al., submitted).

While in yeast and animals the exocyst subunits are typically encoded by a single gene, in land plants they are usually duplicated or even multiplicated (Eliáš et al., 2003; Cvrčková et al., 2012), with extreme gene proliferation in the case of the EXO70 subunit. For example, the genome of Physcomitrella patens encodes 13 EXO70 paralogs, Oryza sativa 47 paralogs and Arabidopsis thaliana 23 paralogs, which can be divided into three clades of ancestral land plant origin, termed EXO70.1, EXO70.2 and EXO70.3 (Cvrčková 
et al., 2012; Rawat et al., 2017; Žárský et al., 2019). This plant-specific multiplicity of EXO70 suggests not only functional specialization in different plant tissues or cell types, but also a presence of several variants of the exocyst complex in the same cell (Žárský et al., 2013). Indeed, some EXO70.2 and EXO70.3 clade members contribute to conventional secretion in specific cell types, while several other isoforms acquired functions outside the conventional exocytosis pathway, acting in exocyst subcomplexes or even independently of the exocyst complex in some cases (Kulich et al., 2013; Kulich et al., 2015; Zhang et al., 2015; Hong et al., 2016; Pečenková et al., 2017; Synek et al., 2017). On the contrary, the Arabidopsis EXO70.1 isoform EXO70A1, highly expressed in most sporophytic tissues, is the main housekeeping EXO70 subunit participating in conventional exocytosis (Synek et al., 2006; Fendrych et al., 2010; Drdová et al., 2013). However, since EXO70A1 is not expressed in pollen, the main EXO70.1 paralog functioning in pollen remained experimentally uncharacterized until now.

The importance of the exocyst for pollen tube germination and growth is well documented. Homozygous Arabidopsis mutants in SEC5a/b, SEC6, SEC8, and SEC15a typically produce short and wide pollen tubes with compromised polarity, resulting in a male-specific transmission defect (Cole et al., 2005; Hála et al., 2008), and sec3a mutants cannot produce pollen tubes at all (Bloch et al., 2016). Transcriptomic and proteomic analyses documented high transcription of the EXOTOA2,C1,C2, F1, H3, H5, and H6 paralogs in Arabidopsis pollen, while EXO70C2, C1, and A2 were the most abundant in Arabidopsis pollen proteome (Grobei et al., 2009, Synek et al., 2017). The two closely related EXO70C paralogs from the EXO70.2 clade participate in the control of optimal pollen tube tip growth, probably via its regulatory function outside the exocyst complex (Synek et al., 2017). On the other hand, the clade EXO70.1 member EXO70A2 is the evolutionarily closest paralog to EXO70A1, the main sporophytic housekeeping isoform (Cvrčková et al., 2012), and also interacts with the same exocyst subunits as EXO70A1 (Synek et al., 2017). Therefore, EXO70A2 represents the best candidate for the main conventional EXO70 isoform in pollen functioning as a part of the exocyst complex in the regulation of polarized exocytosis.

In this report, we document that disruption of EXO70A2 in Arabidopsis affects pollen maturation, significantly reduces pollen germination efficiency and causes a severe pollen-specific transmission defect of the mutant allele due to impaired pollen tube growth. In growing pollen tubes, GFP:EXO70A2 localizes, similarly to other exocyst subunits, to the very apical domain in the tip characterized by intensive exocytosis. Ectopic expression of EXO70A2 could substitute for the EXO70A1 function in the sporophyte, indicating functional redundancy of these two closely related isoforms. Phylogenetic and expression analysis of EXO70.1 genes revealed independent duplications of EXO70A in monocots and dicots and showed that the dicot EXO70A2 clade contains predominantly pollen-specific isoforms. We thus conclude that Arabidopsis EXO70A2 is critical for the pollen function and that, analogous to EXO70A1 in the sporophyte, EXO70A2 is the main EXO70 isoform functioning as a subunit of the exocyst complex in conventional secretion in Arabidopsis pollen.

\section{RESULTS}

\section{EXO70A2 is a member of a dicot pollen-expressed EX070.1 clade}

To clarify the evolutionary relationships among EXO70A paralogs, which comprise the sole family of the ancestral EXO70.1 clade (Synek et al. 2006; Cvrčková et al. 2012; Rawat et al. 2017), we performed a detailed phylogenetic analysis of available predicted protein sequences of this clade members from 19 plant species covering a broad range of land plant diversity including liverworts, mosses, lycophytes, gymnosperms, the basal angiosperm Amborella trichopoda, grasses as representatives of monocots, and dicots including representatives of both asterids and rosids (sequences are listed in Supplemental File S1). The sole EXO70 of the charophyte Klebsormidium flaccidum has been also included as an outgroup. The resulting phylogenetic tree (Fig. 1) clearly indicates that the EXO70A duplication took place independently 
in basal dicots and in monocots (or at least in grasses), resulting in both dicots and grasses possessing representatives of two EXO70A clades that cannot be considered orthologous between the two angiosperm lineages. The Arabidopsis EXO70A1 and EXO70A2 paralogs map into different dicot EXO70A clades, while the third, EXO70A3, is closely related to EXO70A2 and resulted from a recent gene duplication that may have been restricted to Brassicales, or even to some subgroup thereof.

For selected representatives of both monocots and dicots, transcript level data for the analyzed genes were extracted from public databases and mapped onto the phylogenetic tree (Fig. 1). The results document clade-specific distinct expression patterns in both dicots and grasses. In dicots, members of the clade containing Arabidopsis EXO70A1 usually exhibit high expression levels in sporophytic vegetative organs with low or no expression in pollen, while a complementary pattern, i.e. high transcript level in pollen and lower to no expression in the sporophyte, is typical for the clade containing EXO70A2. Also in the grasses one of the two clades contains mostly genes with either ubiquitous or predominantly sporophytic expression, while most members of the other exhibit enhanced, though usually not exclusive, expression in the male gametophyte.

\section{Preparation and characterization of a loss-of-function exo70a2 allele using the CRISPR/Cas9 system} In order to investigate the EXO7OA2 function in planta we employed Arabidopsis mutants. At the time of this study, T-DNA insertion null mutants in EXO7OA2 (At5g52340) were not available. One of the two publicly available lines, GABI_824D06 (exo70a2-1) with the insertion located in 5' UTR, exhibited EXO70A2 overexpression (Synek et al., 2017), and the another, FLAG_264F01 (exo70a2-2) could not be confirmed in our hands. We thus employed the CRISPR/Cas9 system (Wang et al., 2015) to generate a loss-offunction exo70a2 mutant in the Arabidopsis Col-0 background, and obtained a mutant line (exo70a2-3) bearing a 13-bp insertion in the fifth exon of the EXO70A2 gene, causing a frameshift in the CDS (Supplemental Fig. S1A). This mutation probably affected the EXO70A2 RNA stability or processing, because the EXO7OA2 transcript level was reduced to less than half in the exo70a2-3 mutant as evaluated by semi-quantitative RT-PCR in flower material (Supplemental Fig. S1B).

Plants homozygous for the CRISPR-generated insertion, hereafter referred to as exo70a2 only, were then subjected to phenotype analysis and compared to their wild-type siblings. To address whether EXO70A2 might play some role in the sporophyte, despite its minimal expression in sporophytic tissues (Synek et al., 2006; www.Genevestigator.com - Hruz et al., 2008), we inspected the morphology of the mutant and WT plants and evaluated two basic morphological parameters. Primary root length of 7 days old seedlings grown on vertical agar was comparable between exo70a2 and WT (Supplemental Fig. S1C, D). Similarly, 5 weeks old exo70a2 and WT plants showed no differences in the plant height and general plant morphology (Supplemental Fig. S1E, F). In conclusion, the sporophyte of exo70a2 homozygotes was not affected by the mutation, consistent with EXO70A2 being a pollen-specific EXO70 isoform.

\section{The exo70a2 mutant shows a pollen-specific transmission defect}

We next analyzed the transmission of the exo70a2 mutant allele through pollen. Based on PCR genotyping, the progeny of heterozygous exo70a2 mutant plants showed significantly reduced portion of mutant homozygotes in comparison to the normal Mendelian ratio (only $15 \%$ of mutant homozygotes were detected), pointing to a severe transmission defect of this mutant allele (Tab. 1).

To determine whether the transmission defect was male- or female-specific, we performed reciprocal crossing of exo70a2 heterozygotes to WT plants and inspected the frequency of WT and heterozygous progeny. When exo70a2 heterozygous plants were used as pollen donors, only $17 \%$ of the progeny were heterozygous for the exo70a2 allele instead of expected $50 \%$. However, when they were used as pollen recipients, $54 \%$ of the progeny was heterozygous, indicating that the transmission defect was male-specific (Tab. 2). 
Table 1. Segregation of the heterozygous exo70a2 mutant.

\begin{tabular}{lllllll}
\hline & $+/+$ & $+/-$ & $-/-$ & $n$ & $x 2$ & $P$ \\
\hline Expected & $25.0 \%$ & $50.0 \%$ & $25.0 \%$ & & & \\
exo70a2 & $38.3 \%$ & $46.7 \%$ & $15.0 \%$ & 107 & 12.14 & 0.002 \\
\hline
\end{tabular}

+/+, wild-type plants; +/-, heterozygous mutants; -/-, homozygous mutants

Table 2. Reciprocal crossing of the exo70a2 mutant and wild type (Col-0).

\begin{tabular}{lllllll}
\hline \multicolumn{5}{c}{ Pollen donor +/-; Pollen recipient, $+/+$} \\
& $+/+$ & $+/-$ & $\mathrm{n}^{*}$ & $\mathrm{X}^{2}$ & $P$ \\
\hline Expected & $50.0 \%$ & $50.0 \%$ & & & \\
exo70a2 & $82.6 \%$ & $17.4 \%$ & 69 & 29.35 & $<0.001$ \\
\hline
\end{tabular}

\begin{tabular}{lcccccc}
\hline \multicolumn{5}{c}{ Pollen donor + ++; Pollen recipient +/- } \\
& $+/+$ & $+/-$ & $\mathrm{n}^{*}$ & $\mathrm{X}^{2}$ & $P$ \\
\hline Expected & $50.0 \%$ & $50.0 \%$ & & & \\
exo70a2 & $46.2 \%$ & $54.8 \%$ & 93 & 0.53 & 0.47 \\
\hline
\end{tabular}

$+/+$, wild-type plants; +/-, heterozygous mutants

${ }^{*}$ at least 6 independent crossings were prepared

\section{Pollen maturation, germination and pollen tube growth are compromised in the exo70a2 mutant}

To investigate the nature of the pollen-specific transmission defect, we inspected several phases of pollen development, including pollen germination and pollen tube growth in exo70a2 and WT plants.

We observed four times higher percentage of non-viable pollen grains at the mature pollen stage using Alexander staining, indicating a significant difference (WT: 3.4\%, $n=179$; exo70a2: 11.5\%, $n=157$; Chi-square test $p_{-}$value $=0.004$ ) (Supplemental Fig. S2A). However, DAPI staining of pollen nuclei demonstrated that exo70a2 pollen grains could normally reach the tricellular developmental stage. This suggests that the observed defect occurred in the maturation phase (Supplemental Fig. S2B).

We then conducted in vitro germination assay on pollen samples harvested from homozygous and heterozygous exo70a2 mutants as well as from a WT control. While the control pollen started to efficiently germinate within two hours after imbibition, the pollen from exo70a2 homozygotes germinated not 
sooner than only eight hours after the imbibition (Fig. 2A, B). Even after 20 hours the exo70a2 pollen still showed 8-fold lower germination efficiency than WT pollen. Since high concentrations of extracellular reactive oxygen species (ROS) were previously reported at the onset of the pollen germination process (Smirnova et al., 2014), we analyzed the ROS production in WT and exo70a2 pollen grains 20 min after imbibition using histochemical staining of superoxide anion radicals by nitroblue tetrazolium (NBT). The ROS levels were dramatically reduced in mutant pollen grains, indicating their different physiological status (Fig. 2C, D).

To find out whether the mutation only affects pollen tube emergence or also its subsequent elongation, we analyzed pollen tube lengths 20 hours after imbibition in vitro. Indeed, in this experiment, exo70a2 pollen tubes were dramatically shorter than WT ones (Fig. 2A, E). Subsequent analysis of growth dynamics of individual pollen tubes revealed that the averaged maximal growth rate of exo70a2 pollen tubes was 6 times lower in our conditions than that of their WT counterparts (exo70A2: $0.39 \pm 0.08$ $\mu \mathrm{m} \cdot \mathrm{min}^{-1}, \mathrm{WT}: 2.42 \pm 0.37 \mu \mathrm{m} \cdot \mathrm{min}^{-1}, \mathrm{n}>12$; mean $\pm \mathrm{SD}$; Student's t-test $\mathrm{p} \_$value $\left.<0.0001\right)$. Thus, exo70a2 pollen displays both delayed germination and reduced pollen tube growth rate. Interestingly, an inspection of the exo70a2 pollen tube elongation in vivo in self-pollinated pistils showed less dramatic, yet significant, defect in pollen tube growth when compared to WT (Fig. 3). This can be explained by the presence of chemical signals promoting pollen tube growth in a dose-dependent manner produced by the transmitting tract tissues in pistils (Johnson and Preuss 2002; Vogler et al., 2014).

Taken together, the loss of functional EXO70A2 affects not only pollen tube growth but also pollen maturation and germination. These processes are linked to polarized exocytosis, and similar defects have been observed in other exocyst mutants (Cole et al., 2005; Hála et al., 2008; Bloch et al., 2016).

\section{Slow growing exo70a2 pollen tubes display morphological defects without major changes in the cell wall composition}

In the in vitro germination experiments, we noticed that exo70a2 pollen tubes had grown straight, without any branching, but with about $50 \%$ wider diameter than WT ones (Fig. 4). This morphological defect suggests that secretion is delocalized in the apex of exo70a2 pollen tubes, which is consistent with the canonical exocyst function in polarized exocytosis in the tip growth.

Since the exocyst plays an important part in polarized exocytosis, which, in turn, is crucial for cell wall construction, we tested whether the pollen tube growth defect in exo70a 2 might be due to altered cell wall biogenesis. Therefore, we analyzed the distribution of dominant cell wall components in growing pollen tubes in vitro. Nevertheless, pectins showed normal distribution after propidium iodide staining, and cellulose and callose deposition visualized by calcofluor white and aniline blue staining, respectively, only extended more toward the tip in exo70a2 pollen tubes, which could be explained by their much lower growth rate (Fig. 5).

\section{GFP:EXO70A2 complements the exo70A2 mutation and localizes to the PM in pollen tube tips}

For further functional characterization of EXO70A2, we prepared a GFP-tagged variant of EXO70A2 expressed under its native promoter. The pEXO70A2::GFP:EXO7OA2 construct was introduced into exo70a2 heterozygous plants in order to analyze the EXO70A2 localization and functionality in mutant and WT pollen. The expression of pEXO7OA2::GFP:EXO7OA2 complemented the EXO7OA2 disruption: The percentage of non-viable pollen grains in freshly open flowers was similar to WT (complemented line: $3.9 \%, n=231$; WT: $3.4 \%, n=179$; Chi-square test $p_{\text {_value }}=0.772$ ). The ability of exo70a2 pollen to germinate in vitro without any delay was restored (Fig. 6A, B; Supplemental Fig. S3), the pollen tube growth defect was reverted to normal (Fig. 6C) and also the pollen tube width was similar to WT tubes (mean \pm SD; complemented line: $6.20 \pm 0.44 \mu \mathrm{m}$; WT: $6.37 \pm 0.72 \mu \mathrm{m}, \mathrm{n}>32$; Student's test $\mathrm{p} \_$value < 0.2631). These observations confirm that the reported defects in exo70a2 pollen were due to the disruption of the EXOTOA2 gene. 
While seedlings and mature plant organs exhibited no fluorescence signal of GFP:EXO70A2 (not shown), we clearly detected GFP:EXO70A2 in pollen grains and pollen tube tips (Fig. 7). In the unicellular pollen stage, GFP:EXO70A2 was found mostly in the nucleus; in the bicellular pollen stage, it was partially re-localized to the cytoplasm; and in the later stages, it was evenly distributed throughout the cytoplasm, but completely excluded from nuclei (Fig. 7A). Importantly, in complemented exo70a2 pollen tubes, GFP:EXO70A2 displayed a highly polarized localization along the PM in the apical domain of growing pollen tube tips with a minor portion in the cytoplasm (Fig. 7B), which is a typical pattern for exocyst subunits (Synek et al., 2017). In the WT background, however, the polar PM localization was absent and GFP:EXO70A2 was distributed entirely in the cytoplasm of pollen tubes (Fig. 7C), which could be explained by a competition of the native and GFP-tagged EXO70A2 variants for binding into the exocyst complex as observed previously in tobacco pollen tubes (Sekereš et al., 2017), and also in mammalian cells (Matern et al. 2001).

\section{EXO70A2 can rescue the EXO70A1 disruption in the sporophyte}

We were interested what is the degree of functional specialization versus redundancy of EXO70A1 and EXO70A2 paralogs implied by their close sequence similarity (see Fig. 1). In order to address it experimentally we expressed EXO70A2 and EXO7OA1, respectively, under the control of the EXO70A1 promoter in the exo70a1 mutant background and analyzed EXO70A2 localization and functionality in the sporophyte. The exo70a1 mutant was earlier shown to exhibit pleiotropic morphological defects, including retarded polar growth of root hairs, a loss of apical dominance, dwarfish stature and sterility (Synek et al., 2006). Moreover, GFP:EXO70A1 fully complemented the exo70a1 loss-of-function allele and localized at the PM, with special enrichment at the outer lateral domain in root epidermal cells (Drdová et al., 2013; Fendrych et al., 2013).

We found that the expression of pEXO7OA1::GFP:EXO7OA2 rescued the morphological defects of exo70a1 mutant plants similarly to $P E X O 70 A 1:: G F P: E X O 70 A 1$, including plant height of 7-week-old plants (Fig. 8A, B). Moreover, the GFP:EXO70A2 localization was identical to GFP:EXO70A1 in the root epidermal cells, suggesting that EXO70A1 and EXO70A2 paralogs are functionally redundant in the sporophyte (Fig. $8 \mathrm{C})$.

\section{DISCUSSION}

In order to deliver sperm cells to ovules and achieve successful fertilization, highly efficient secretory machinery is essential to sustain the fast pollen tube growth. Targeting of secretory vesicles to a very small PM area in the growing pollen tube tip requires, among other regulators, also the vesicletethering exocyst complex. The crucial role of the exocyst in the tip growth was previously evidenced by the aberrant morphology of pollen tubes in Arabidopsis mutants deficient in core exocyst subunits (Cole et al., 2005; Hála et al., 2008). However, the main EX070.1 isoform contributing to the conventional secretory function of the exocyst complex in pollen awaited experimental characterization until now.

We previously described that EXO70.2 clade members, EXO70C2 and EXO70C1, are not stable subunits of the exocyst complex (Synek et al., 2017), leaving EXO70A2 the best candidate for the main housekeeping EXO70 isoform in pollen. First, EXO70A2 is the closest paralog to EXO70A1 - the main EXO70 isoform in the Arabidopsis sporophyte - and these two share $72 \%$ sequence identity at the protein level. Second, EXO70A2 also physically interacted with three other exocyst subunits (SEC3a, SEC10b and EXO84b), similarly to EXO70A1 (Hála et al., 2008; Fendrych et al., 2010; Synek et al., 2017). Third, the expression patterns of EXO7OA1 and EXO7OA2 alternate between the sporophyte-specific and pollenspecific, respectively. This is in agreement with the hypothesis suggesting that after a whole-genome duplication in plants, genes directly linked to cell polarity tend to diverge in their expression patterns to 
sporophyte-specific and to those enriched in tip-growing cells, especially in the male gametophyte (De Smet et al., 2017). In general, about $10 \%$ of essential housekeeping genes evolved their pollen specific paralogs to sustain the specific requirements for tip growth (Becker and Feijó, 2007).

Indeed, in course of the extraordinary diversification and functional specialization of the large EXO70 family in angiosperms (Cvrčková et al., 2012), the EXO70A duplication to sporophyte- and male gametophyte-specific paralogs occurred independently in monocots and dicots. In some plant taxonomic groups, each of them further duplicated, generating, for example, EXO70A2 and EXO70A3 in Brassicaceae, and EXO70C1a and EXO70C1b in Solanaceae (Sekereš et al., 2017). An analogous situation, i.e. the presence of pollen-optimized paralogs, has been documented in the case of SEC3, SEC15 and EXO84 exocyst subunits in Arabidopsis, where SEC3a, SEC15a and EXO84a are pollen-specific, while their siblings are active in the sporophyte (Bloch et al., 2016; Synek et al., 2017; Batystová et al., submitted). Analogically to our observation that the artificial EXO70A2 expression in the sporophyte could complement the sporophytic defects of exo70a1 mutants, SEC15a, the main SEC15 isoform in pollen, could substitute for the function of SEC15b in the sporophyte, although SEC15a and SEC15b share mere $47 \%$ sequence identity at the protein level (Batystová et al., submitted). It is tempting to speculate that exocyst complexes with different subunit composition may bind vesicles with distinct secretory cargos and deliver them to special PM subdomains (due to different EXO70-membrane interactions, see Žárský et al., 2009; Sekereš et al., 2017; Kubátová et al. 2019). In contrast, SEC10a and SEC10b represent a very recent duplication without any signs of expression or functional diversification (Vukašinović et al., 2014).

Based on the phenotypic deviations of exo70a2 mutants, we documented that EXO70A2 participates in pollen maturation, germination, and pollen tube tip growth. Also mutants in two other exocyst subunits, $\sec 3 a$ and $\sec 8$, exhibited severe defects in their germination efficiency (Bloch et al., 2016; Cole et al., 2005). One of the crucial regulatory modules in pollen germination and pollen tube growth is the ROS production (Potocký et al., 2007; Potocký et al., 2012; Smirnova et al., 2014). Two Arabidopsis pollen-specific NADPH oxidases, RBOHH and RBOHJ, localized at the PM are necessary for ROS accumulation in pollen grain cell wall upon pollination and later in pollen tube tips. Double mutants in these genes exhibited much lower ROS level resulting in collapsing pollen tubes (Lassig et al., 2014; Kaya et al., 2015). Interestingly, data of Smirnova et al. (2014) suggest that targeted production of extracellular ROS is required in order to change mechanical properties of intine pollen grain cell wall to ensure germination. Since we observed a significant decrease in ROS production in exo70a2 germinating pollen grains, we suggest that the pollen exocyst may function in the delivery of NADPH oxidases to specific sites at the PM during germination.

Following pollen germination, an elongating pollen tube requires precisely regulated secretory machinery to support the intensive tip growth. Mutants in several core exocyst subunits $(\sec 5 a / b$, sec6, $\sec 8$, sec $15 a$ ) generate extremely short and wide pollen tubes with drastically reduced fertilization capacity similarly to exo70a2 described here (Hála et al., 2008). This pollen tube phenotype is most likely caused by an inefficient targeting of secretory vesicles during the tip growth, because the deposition of major cell wall components was otherwise normal. In contrast, the mutant in EXO70C2, a member of the EXO70.2 clade, exhibited very different phenotype of mutant pollen tubes, caused by impaired regulation of growth rate, which pointed to a diverged function of EXO70C2 (and its close paralog EXO70C1) independent of the conventional exocyst function (Synek et al., 2017). Furthermore, EXO70A2 showed the PM localization identical to core exocyst subunits, SEC8, SEC10a and SEC15a in growing pollen tube tips in Arabidopsis (Synek et al., 2017; Batystová et al., submitted). At the molecular level, EXO70A2 probably mediates the exocyst interaction with the PM similarly to Exo70 in yeast and mammalian cells (Boyd et al., 2004; He et al., 2007; Pleskot et al., 2015). This notion is supported by our observation that the N-terminally truncated SEC3a subunit in Arabidopsis pollen was unable to interact with the PM, but still localized to the apical PM in growing pollen tube tips as a part of the complex (Bloch et al., 2016). 
Although the transmission efficiency of the exo70a2 mutant allele was heavily impaired, the mutation did not cause a complete transmission defect like that observed in exocyst loss-of-function mutants in the core exocyst subunits (sec6, sec8, sec15a) (Hála et al., 2008). This indicates that some other EXO70 isoform(s) could provide a partially overlapping function to EXO70A2; possible candidates include $\mathrm{EXO} 70 \mathrm{H} 3, \mathrm{H} 5$ and $\mathrm{H} 6$. However, their abundance in the pollen proteome is most likely very low (Synek et al., 2017) and their relevance thus would have to be proved experimentally. Alternatively, EXO7OA1 might be activated when EXO7OA2 is disrupted, despite the fact that EXO7OA1 is normally not expressed in pollen (Synek et al., 2006; www.Genevestigator.com - Hruz et al., 2008). In general, we propose that the other six EXO70 isoforms expressed in pollen adopted specific functions with no or only partial functional overlap with EXO70A2. In addition, their activities might be restricted to certain stages of pollen development or specific endomembrane domains.

Proper cell wall deposition is essential for efficient pollen germination and pollen tube growth (Chebli et al., 2012; Leroux et al., 2015; MacAlister et al., 2016). Since the exocyst is responsible for efficient polarized delivery of cell wall materials and cell wall modifying enzymes, it is not surprising that exo70a 2 mutants exhibit defects in both pollen germination and pollen tube growth. Similar phenotypes were reported also for several Arabidopsis mutants in pollen-specific pectin methylesterases (PME). Notably, the homozygous pme48 mutant showed a significant delay in the pollen grain germination and produced significantly wider pollen tubes (Leroux et al., 2015). Loss of PPME1 function lead to reduced growth rate and increased width of pollen tubes (Tian et al., 2006). The disruption of PME VANGUARD1 resulted in impaired pollen tube elongation in vivo and bursting of pollen tubes in vitro (Jiang et al., 2005). The excessive amount of methylesterified pectins in the intine cell wall of pollen grains and at the pollen tube tip in PME mutants, reduced the $\mathrm{Ca}^{2+}$ binding and formation of pectate gel, thus decreasing the rigidity of the cell wall (Leroux et al., 2015). Moreover, higher amount of hydrophobic methylesterified pectins in PME mutants also decreased the rate of pollen grain water uptake during imbibition, affecting the germination efficiency (Leroux et al., 2015). Other cell modifying enzymes are also important for pollen tube tip growth. For example, mutants in hydroxyproline O-arabinosyltransferases, HPAT1 and HPAT3, involved in modification of cell wall-associated extensins, exhibited a similar phenotype to exo70a2 regarding their defect in pollen tube elongation (MacAlister et al., 2016). Higher percentage of non-viable pollen grains and delayed germination in exo70a2 could be also explained by mislocalization or inefficient delivery of some cell wall modifying enzymes (or other cell wall components) whose delivery depends on EXO70A2-containing exocyst complex during pollen maturation and germination.

In summary, we conclude that the duplication of EXOTOA genes to sporophyte- and male gametophyte-specific paralogs occurred independently in monocots and dicots. We proved that among seven EXO70 isoforms expressed in Arabidopsis male gametophyte EXO70A2 is indeed the main conventional EXO70 isoform contributing to the canonical exocyst function in polarized secretion analogically to its sibling, EXO70A1, in the sporophyte. However, EXO70A2 seems to play several roles in the male gametophyte, because it is important not only for the pollen tube tip growth, but also for the previous phases - pollen grain maturation and germination. Importantly, despite the deep evolutionary split, EXO70A2 still keeps the ability to fully substitute for the EXO70A1 function in the sporophyte. Overall, we characterized a new molecular component critical for pollen function and therefore for efficient plant sexual reproduction.

\section{MATERIALS AND METHODS}

\section{Phylogenetic and expression analysis}

To perform the phylogenetic analysis, a set of EXO70.1 clade sequences from our previous studies (Cvrčková et al., 2012; Rawat et al., 2017) has been updated according to their newest database status 
and homologs from additional plant species were obtained by BLASTP searches of the GenBank, Phytozome (Goodstein et al., 2012) and GDR (www.rosaceae.org) databases using Arabidopsis EXO70A1 (At5g03540) and EXO70A2 (At5g52340) sequences as a query. The full list of sequences is provided in Supplemental File S1. Protein sequence alignment was constructed using the MAFFT E-INS-I algorithm (Katoh and Standley, 2013) in Jalview software (Waterhouse et al., 2009). Gaps and non-conserved regions were then manually deleted from the alignment, giving the matrix of 56 taxa and 546 positions. Bayesian phylogeny inference was performed using MrBayes software (Ronquist et al., 2012) with a WAG amino acid model, where the analysis was performed in four runs with four chains and 500,000 generations, and trees were sampled every 100 generations. All four runs asymptotically approached the same stationarity after the first 125,000 generations, which were omitted from the final analysis. Maximum-likelihood phylogeny was performed in Phyml software (Guindon et al., 2010) using the LG matrix, $\mathrm{p}$-corrected for among-site rate variation with four rate site categories plus a category for invariable sites, with all parameters estimated from the data model to build the phylogenetic tree. Bootstrap analysis (500 replicates) was performed to estimate the probability of maximum-likelihood tree topology.

Analysis of the EXO70.1 paralogues expression in male gametophyte and sporophytic tissues was performed using the CoNekT (Proost and Mutwil, 2018) and GEO (Barrett et al., 2013) tools. Additional anther/pollen RNA-seq expression data for sorghum, Arabidopsis, poplar and tobacco were obtained from (Davidson et al., 2012; Loraine et al., 2013; Zhao et al., 2016; Conze et al., 2017), respectively.

\section{Plant material and growth conditions}

The Arabidopsis exo70a1-2 (SALK_135462; Synek et al., 2006) and exo70a2-1 (GABI_824D06; Synek et al., 2017) lines were described previously. The exo70a2-2 line (FLAG_264F01) was obtained from INRA. Genotypes of individual plants were always analyzed by PCR genotyping (for primers - see Supplemental Tab. S1).

Seeds were surface sterilized (70\% ethanol for $3 \mathrm{~min}, 20 \%$ commercial bleach for $5 \mathrm{~min}$, rinsed four times with sterile distilled water) and stratified for 2 days at $4^{\circ} \mathrm{C}$. Seeds were germinated on vertical $1 / 2$ MS agar plates (one-half-strength Murashige and Skoog medium [Duchefa Biochemie] supplemented with $1 \%$ sucrose, vitamin mixture, and $1 \%$ plant agar [Duchefa Biochemie]) at $22^{\circ} \mathrm{C}$ under long-day conditions (16 h light/8 $\mathrm{h}$ dark cycles). Seedlings were transferred to turf pellets (Jiffy Products International, Norway) after 8 days and grown until harvest at the same growth conditions.

\section{Preparation of the exo70a2 mutant line}

The new Arabidopsis mutant line (exo70a2-3) was generated using the egg cell-specific promotercontrolled CRISPR/Cas9 technology, employing the pHSE401E vector (Wang et al., 2015). To increase the chance of a mutation, two target single-guide RNAs (sgRNAs) were designed. Flowering Arabidopsis plants of the Col-0 ecotype (NASC collection) were transformed using the Agrobacterium tumefaciens-mediated floral dip (Clough and Bent, 1998). After selection on hygromycin, a mutant line was identified by sequencing of PCR products obtained from the targeted part of the EXO70A2 gene (At5g52340) having a $13 \mathrm{bp}$ insertion in the expected site (...TCGAGCTGCGGTttcatgcgattttGTTGGAACAGAG...). The genotype was then analyzed by PCR genotyping, when one of each pair of primers for the wild-type and mutant allele, respectively, were designed over the CRISPR-modified site (see Supplemental Fig. S1A and Tab. S1 for primers).

\section{Semi-quantitative RT-PCR analysis of the EXO70A2 transcription}

To determine the EXO70A2 transcript level in the CRISPR-modified mutant line, total RNA was extracted from $100 \mathrm{mg}$ of apical parts of primary inflorescences of exo70a2 and WT plants using the RNeasy kit (Qiagen). Prior to reverse transcription, samples were treated by DNase I (New England Biolabs). The cDNA was synthesized using oligo-dT primers, $2 \mu \mathrm{g}$ of total RNA and Transcriptor High Fidelity cDNA Synthesis 
kit (Roche). Transcript abundance was assayed by semi-quantitative PCR for two gene regions - up-stream and down-stream from the CRISPR-generated insertion (see Supplemental Fig. S1) - using EXO7OA2specific primers; the primers were especially checked for potential interference with EXOTOA1 and EXO7OA3 paralogs (Supplemental Tab. S1). ACTIN7-specific primers were used as a quantitative control. The optimal number of PCR cycles was determined empirically.

\section{Analysis of pollen development}

Pollen grains at different developmental stages were collected from a series of flower buds ending with a freshly open flower and used for further analyses of nuclear division and pollen grain viability.

To analyze the developmental progress, pollen grains were stained with 4',6-diamino-2-phenylindole (DAPI) at final concentration $4 \mathrm{\mu g} / \mathrm{ml}$ in PIB buffer $\left(100 \mathrm{mM} \mathrm{Na} 3 \mathrm{PO}_{4}, \mathrm{pH}=7.5 ; 1 \mathrm{mM}\right.$ EDTA; 0.1\% [v/v] Triton X-100) as described by Backues et al. (2010). Images were captured using a Zeiss Axio Imager 2 microscope with an EC Plan-Neofluar 40x/0.75 objective, a filter set for observation of blue fluorescence, DIC optics, and Zeiss Axiocam 506 Color camera.

To inspect the pollen grain viability we used Alexander staining (Alexander, 1969) with following modifications: 10x diluted, $15 \mathrm{~min}$ incubation time at room temperature. Images were captured using a Nikon Eclipse 90i microscope with Plan Apo 10x/0.45 objective, DIC optics, and a Zyla sCMOS camera (Andor). Fully developed non-viable (not stained) pollen grains, but not collapsed underdeveloped grains, were counted.

\section{Germination of Arabidopsis pollen in vitro}

Pollen was germinated on a thin layer of semi-solid pollen germination medium ( $10 \%$ sucrose, $1.5 \%$ lowmelting-point agarose (Agarose-Universal, peqGOLD, VWR), $0.01 \% \mathrm{H}_{3} \mathrm{BO}_{3}, 5 \mathrm{mM} \mathrm{CaCl}, 5 \mathrm{mM} \mathrm{KCl}$ and 1 $\mathrm{mM} \mathrm{MgSO}_{4}$ ) in chambered Lab-Tek II coverglass (Thermo Scientific). Pollen grains from fully opened flowers were spread onto the medium layer, chambers were closed and placed into standard plant growth conditions (see above).

To evaluate the germination efficiency, pollen grains were supposed as germinated when a pollen tube length reached at least one half of pollen grain diameter. For each genotype, 10 samples originating from 4 different plants were observed. Pictures of germinated pollen were taken using Nikon Eclipse 90i microscope with Plan Apo 10x/0.45 objective. Pollen tube lengths were measured in ImageJ and histograms were generated in Microsoft Excel.

\section{ROS staining}

Production of superoxide was determined by its ability to reduce nitroblue tetrazolium (NBT) to formazan precipitate (Rossetti and Bonatti, 2001). Pollen grains were spread on semi-solid pollen germination medium (see above). After 20 min of imbibition it was overlaid with $20 \mu$-drops of NBT diluted in liquid pollen germination medium (final concentration $2 \mathrm{mg} / \mathrm{ml}$ ), incubated for $10 \mathrm{~min}$, and immediately imaged using a Nikon Eclipse 90i microscope with Plan Apo 20x/0.75 objective, excluding DIC filters, and maximum field aperture opening. NBT signal intensity was measured in ImageJ. Values were normalized to nonstained pollen grains and related to maximal staining intensity value across all samples. The experiment was repeated in three replicas with similar results.

\section{Pollen tube morphology and growth rate}

Details of pollen tube morphology were captured using Zeiss Axio Imager 2 microscope with an EC PlanNeofluar 40x/0.75 objective 3 or 16 hours after imbibition for wild type and exo70a2, respectively. The pollen tube growth rate was analyzed using the same microscope but with EC Plan-Neofluar 20x/0.5 objective. Time-lapses at a 2 min interval were recorded for $30 \mathrm{~min}$ (starting $3 \mathrm{~h}$ after imbibition) or for $90 \mathrm{~min}$ (starting $16 \mathrm{~h}$ after imbibition), for WT and exo70a2, respectively. Only pollen tubes longer than 
twice the pollen grain diameter were evaluated, because those already had reached the maximal growth rate.

\section{Callose visualization in Arabidopsis pistils}

Self-pollinated pistils (at least 30 for each genotype) from fully opened flowers were collected and stained with aniline blue according to Mori et al. (2006) and imaged using a Nikon Eclipse 90i microscope with a Plan Apo 4x/0.2 objective and a Zyla sCMOS camera (Andor). Length of the longest pollen tube in every pistil was measured, and recorded values were evaluated statistically using Student's t-test.

\section{Fluorescent staining of cell wall components}

Propidium iodide, Calcofluor White or Aniline Blue was diluted in liquid germination medium and gently applied onto pollen germinated in vitro immediately before imaging. Images were captured using a Zeiss LSM 880 confocal laser scanning microscope with Plan-Apochromat 10x/0.45, Plan-Apochromat 20x/0.8, C-Apochromat 40x/1.2 WI, and C-Apochromat 63x/1.2 WI objectives. Working concentrations, excitation laser wavelengths, and the range of recorded emission wavelengths were as follows: propidium iodide, $30 \mathrm{mM}, 514 \mathrm{~nm}$, and 566-719 nm; Calcofluor White, $1 \mathrm{mg} / \mathrm{ml}, 405 \mathrm{~nm}$, and 410-523 nm; decolorized Aniline Blue, $0.001 \%, 405 \mathrm{~nm}$, and $410-535 \mathrm{~nm}$.

\section{Cloning, complementation assays and imaging of GFP-tagged EXO70A2}

All constructs were prepared using the MultiSite Gateway ${ }^{\circledR}$ (Invitrogen). For preparation of the pEXO7OA2::GFP:EXO7OA2 construct the EXO7OA2 promoter (1,068 bp upstream from the start codon) was subcloned into pENTR 5'-TOPO; the GFP gene in pEN-L1-F-L2 was obtained from VIB in Ghent (Karimi et al., 2007); the EXO70A2 (At5g52340) with the stop codon was subcloned into pDONR P2R-P3 using the Gateway BP clonase (Invitrogen). The three elements were then recombined together into the pB7m34GW destination vector (Karimi et al., 2007) using the Gateway LR clonase (Invitrogen).

For cloning of pEXO7OA1::GFP:EXO7OA2 and pEXO7OA1::GFP:EXO7OA1, the EXO7OA1 promoter (1kb upstream from the start codon) was subcloned into pDONR P4-P1r. The EXO7OA1 was subcloned into pDONR P2R-P3. Then, multi-site reactions were performed to assemble the EXO70A1 promoter, GFP, and EXO70A1 or EXO70A2 into the destination vector pB7m34GW. The insertions in pDONR vectors were sequenced using M13 primers. The final constructs were sequenced using M13 primers and two GFP primers (see Supplemental Tab. S1).

The final pEXO70A2::GFP:EXO70A2 construct was then introduced to exo70a2 heterozygous plants, while $p$ EXO70A1::GFP:EXO7OA1 and pEXO70A1::GFP:EXO70A2 to exo70a1 heterozygous plants, using the Agrobacterium tumefaciens-mediated floral dip method (Clough and Bent, 1998). Transformed seedlings were selected on BASTA. Complementation assays were performed using 3 or more independent transformed lines for each construct.

The subcellular localization of GFP:EXO70A2 in pollen grains, pollen tubes and roots was performed using a Zeiss LSM 880 confocal laser scanning microscope equipped with C-Apochromat $63 \mathrm{x} / 1.2 \mathrm{WI}$. The fluorophore was excitation with a 488-nm laser, and emitted fluorescence was recorded at 493-535 $\mathrm{nm}$. The pollen was germinated as described above and carefully transferred to the microscope $2 \mathrm{~h}$ after imbibition. 


\section{FIGURES}

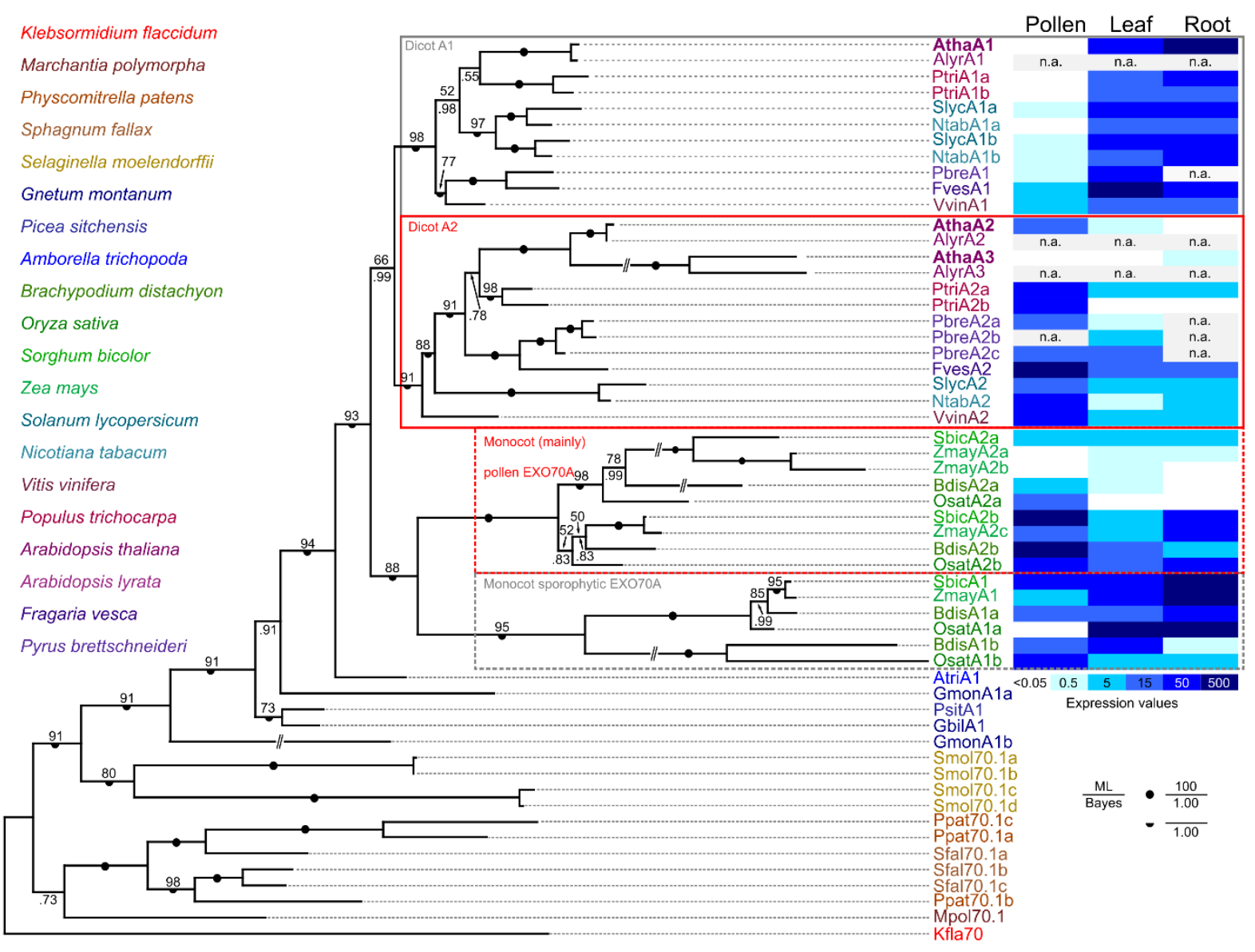

Figure 1. Phylogenetic and expression analysis of the EXO70.1 clade in land plants.

A Bayesian phylogenetic tree of EXO70.1 protein sequences from representative land plants ranging from liverworts (Marchantia polymorpha), mosses (Physcomitrella patens, Sphagnum fallax), lycophytes (Selaginella moelendorffii), gymnosperms (Gnetum sp., Picea sitchensis), the basal angiosperm Amborella trichopoda, grasses (Brachypodium distachyon, Oryza sativa, Sorghum bicolor and Zea mays) as representatives of the monocots, and dicots represented by both asterids (Solanum lycopersicum, Nicotiana tabacum) as well as multiple rosids (Vitis vinifera, Populus trichocarpa, Arabidopsis thaliana and lyrata, Fragaria vesca, Pyrus brettschneideri). The sole EXO70 of the charophyte Klebsormidium flaccidum has been included as an outgroup (for a full list of sequences with accessions see Supplemental File S1). A consistent tree was also obtained by the maximum likelihood method (bootstrap values shown); full support for some branches is denoted by symbols. For selected angiosperm species, a heat map of transcript levels in pollen and sporophytic tissues, as inferred from publicly available transcriptome data, is shown. 
A
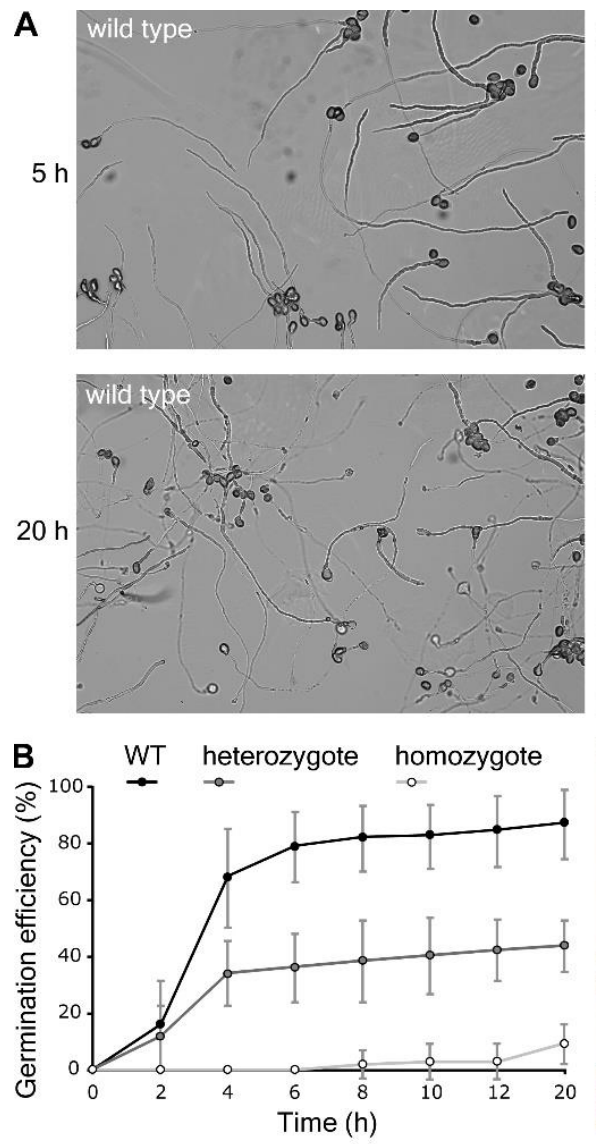

E

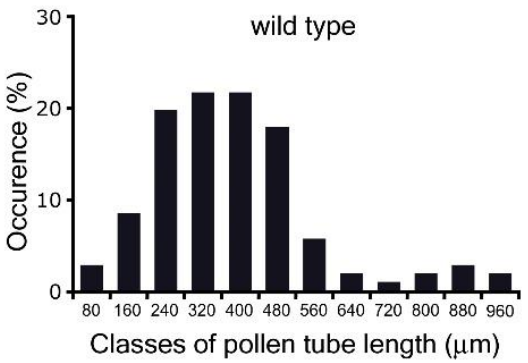

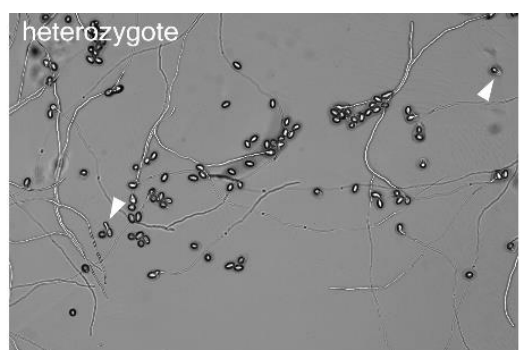
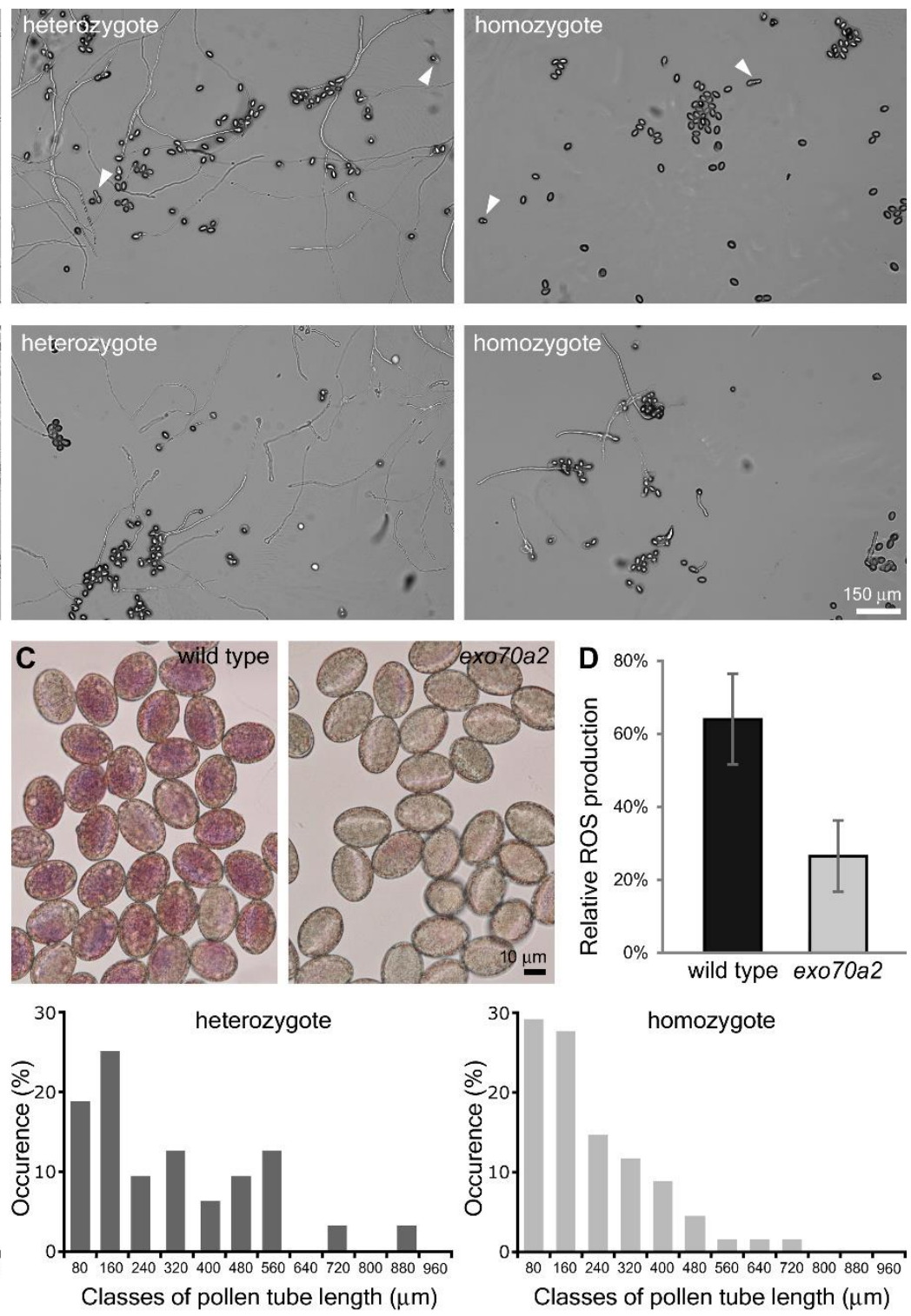

Figure 2. In vitro pollen germination and pollen tube growth defects of exo70a2 mutants.

A) Representative micrographs of in vitro germinated pollen from WT and heterozygous and homozygous exo70a 2 mutant plants $5 \mathrm{~h}$ and $20 \mathrm{~h}$ after imbibition. Arrowheads indicate germinated mutant pollen tubes.

B) Pollen germination efficiency in vitro at different time points (10 samples originating from 3 different plants were evaluated for each genotype, error bars represent SD).

C) ROS production 20 min after pollen imbibition as visualized by NBT staining.

D) Quantification of the ROS staining (in C) shows a significant difference between WT and exo70a2 pollen grains (error bars represent SD, t-test $p_{-}$value $<0.001$ ).

E) Distribution of pollen tube lengths $20 \mathrm{~h}$ after imbibition. More than 100 pollen tubes were evaluated for WT and heterozygous plants, and 50 pollen tubes for the exo70a2 mutant. 
A
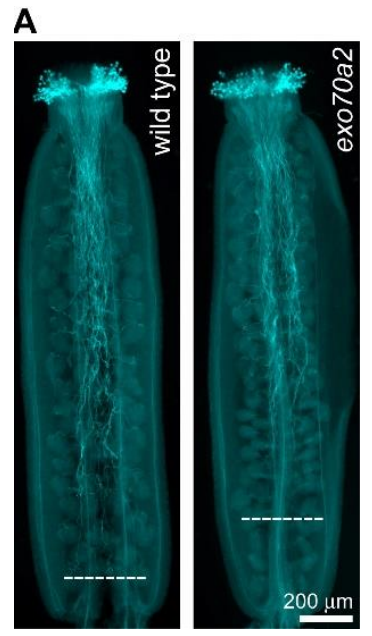

B

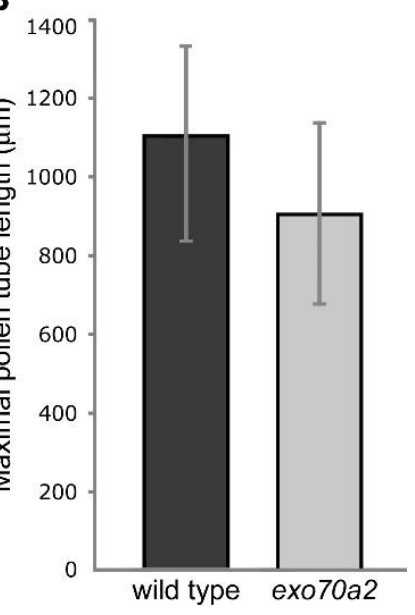

Figure 3. In vivo growth of exo70a2 and WT pollen tubes.

A) Aniline blue staining visualizing callose in pollen tubes in representative self-pollinated pistils from a WT and homozygous exo70a2 plant. Dashed lines indicate the longest pollen tubes.

B) Quantification of maximal pollen tube lengths based on aniline blue staining (at least 30 pistils of each genotype were evaluated, error bars represent SD, Student's t-test $p_{-}$value $<0.01$ ).

Figure 4. Morphology of exo70a2 and WT pollen tubes in vitro.

A) WT and exo70a2 pollen tubes $1.5 \mathrm{~h}$ and $8 \mathrm{~h}$, respectively, after imbibition.

B) Measurement of WT and exo70a2 pollen tube width showed a significant difference ( $n>46$; Student's t-test p_value $<0.0001$ ). 

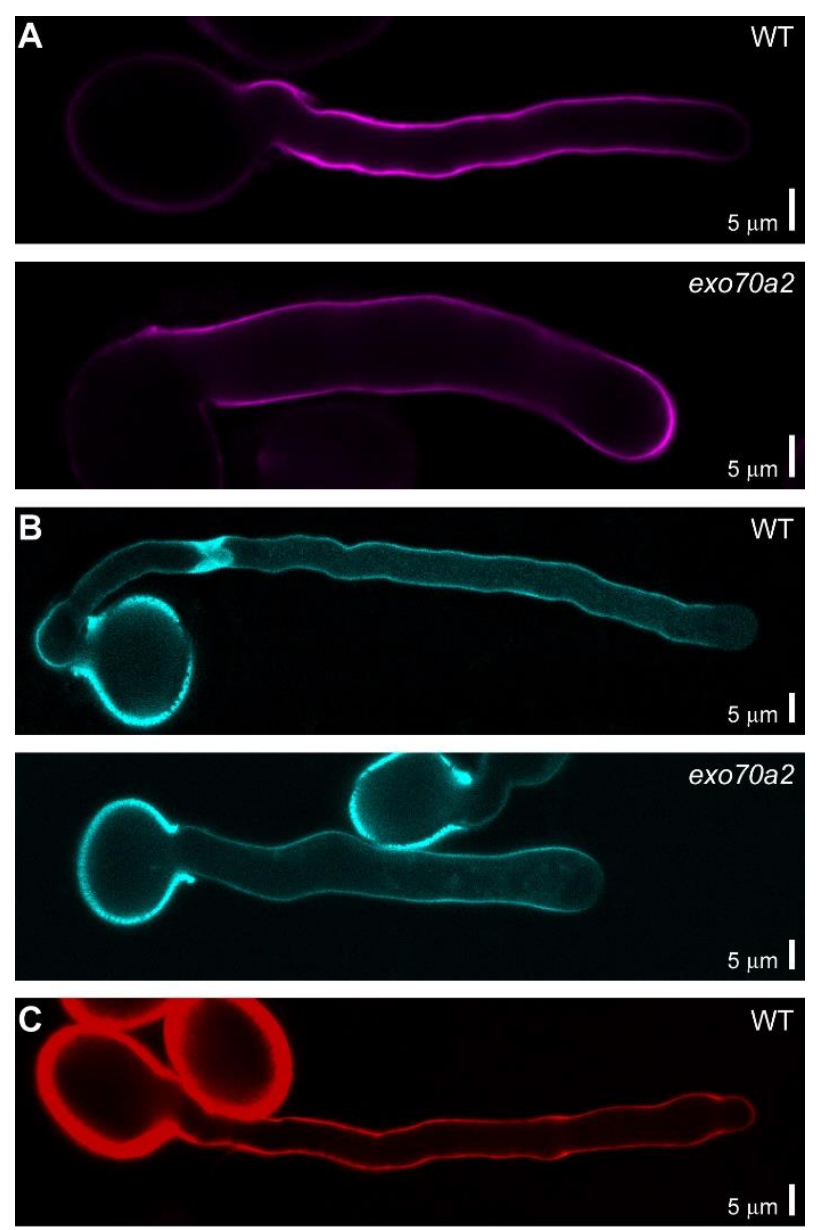

exo70a2

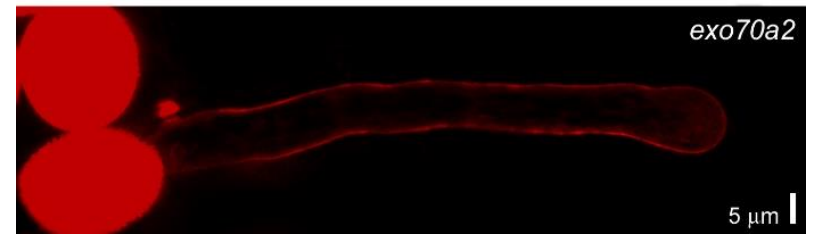

Figure 5. Cell wall deposition in growing WT and exo70a2 pollen tubes in vitro.

A) Visualization of cellulose by calcofluor white staining.

B) Visualization of callose by aniline blue staining.

C) Visualization of pectins by propidium iodide staining. 

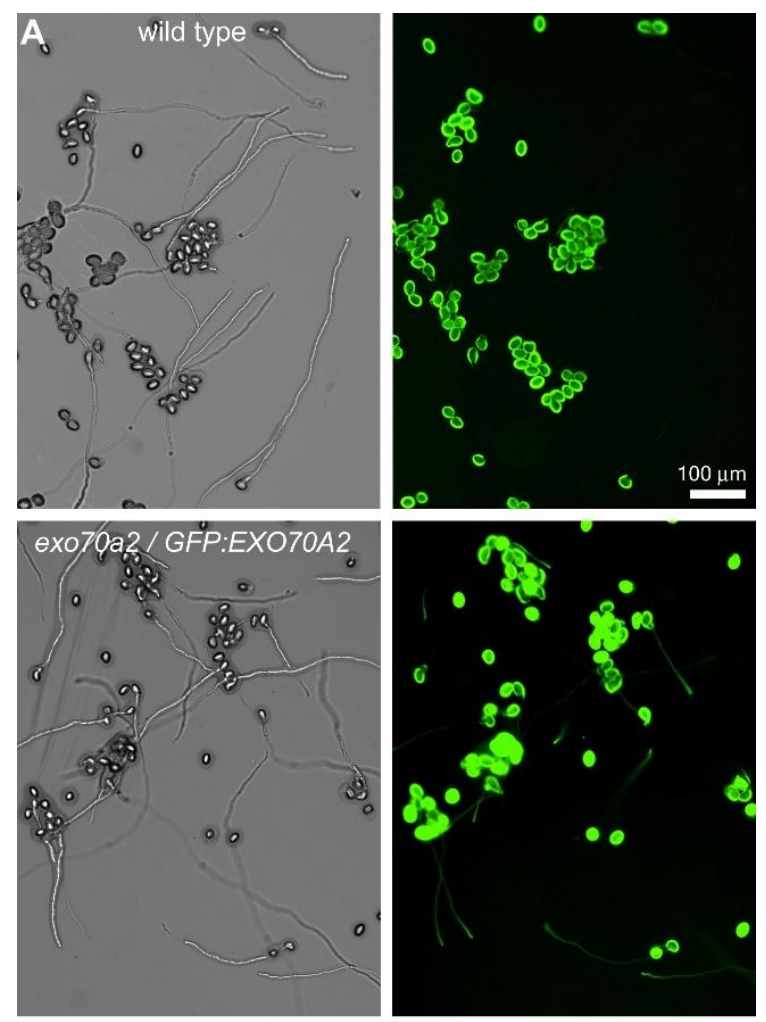

B
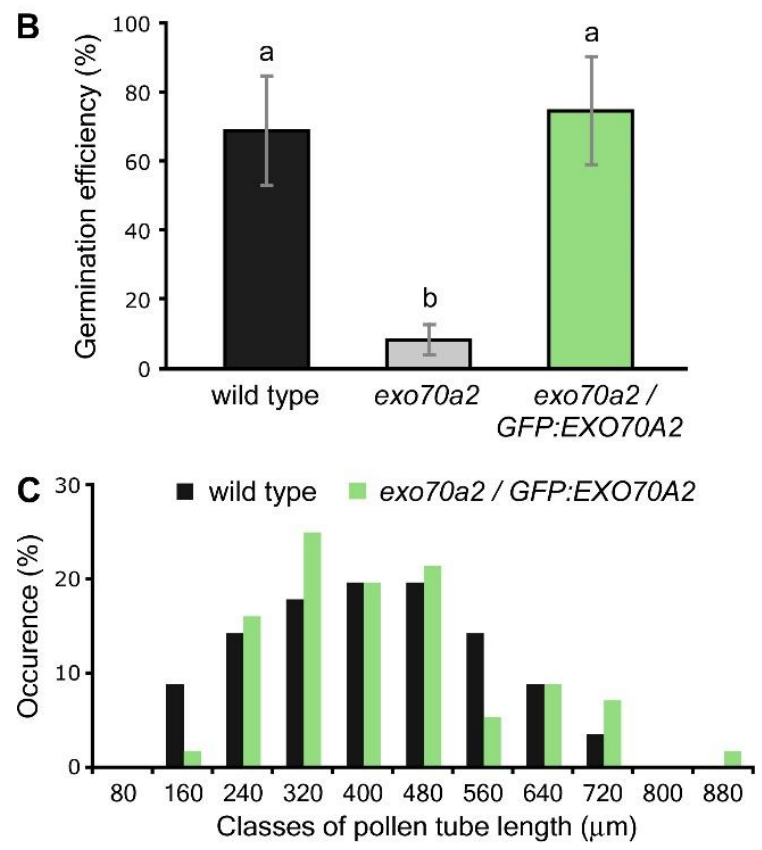

Figure 6. Expression of pEXO7OA2::GFP:EXO70A2 complements the defects of the exo70a2 mutant pollen.

A) Representative micrographs of in vitro germinated pollen from WT and the exo70a2/pEXO70A2::GFP:EXO7OA2 line $20 \mathrm{~h}$ after imbibition.

B) Pollen germination efficiency in vitro $20 \mathrm{~h}$ after imbibition (10 samples originating from 3 different plants were evaluated for each genotype, error bars represent SD). Letters denote statistically different groups evaluated by ANOVA at 0.01 significance level.

C) Distribution of pollen tube lengths $20 \mathrm{~h}$ after imbibition. More than 60 pollen tubes for each genotype were evaluated. 

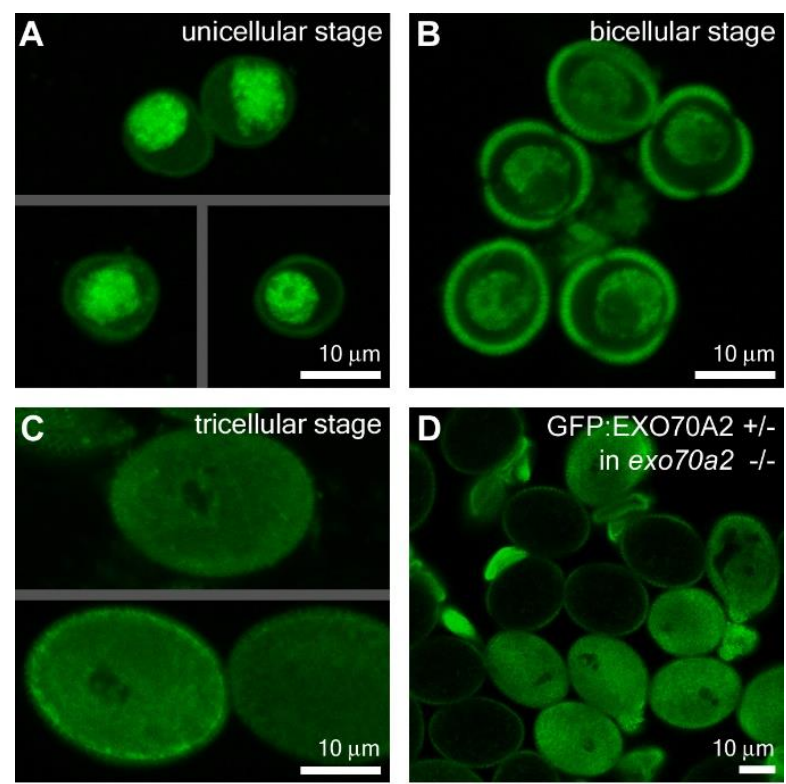

E

GFP:EXO70A2 in exo70a2 -1-
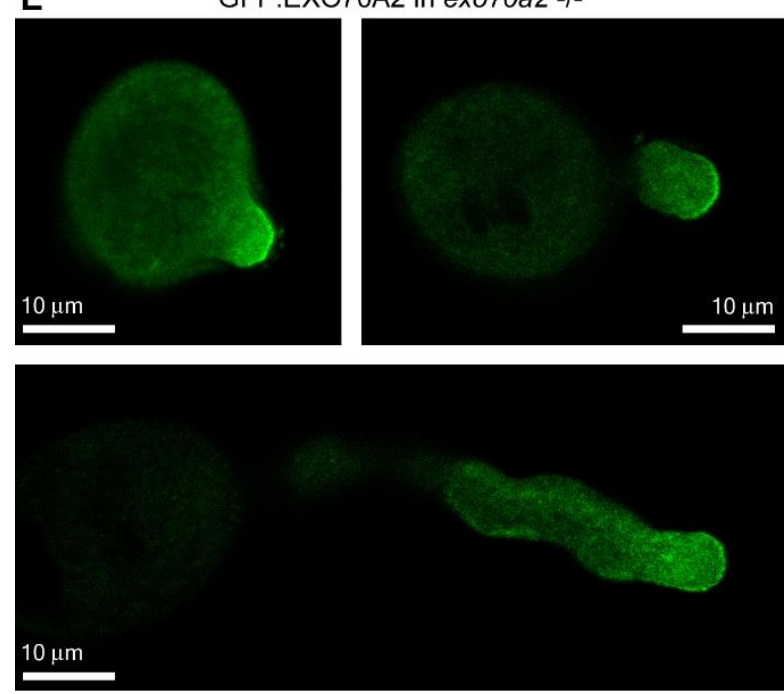

$\mathbf{F}$

GFP:EXO70A2 in WT
Figure 7. Localization of GFP:EXO70A2 expressed under its native promoter in pollen.

A) GFP:EXO70A2 localization in unicellular pollen grains in the exo70a2 background.

B) GFP:EXO70A2 localization in bicellular pollen grains in the exo70a2 background.

C) GFP:EXO70A2 localization in tricellular pollen grains in the exo70a2 background. Note that images in A, B and C were captured at an identical setting, allowing for their quantitative comparison.

D) GFP:EXO70A2 localization in mature exo70a2 pollen grains in a line segregating for the GFP:EXO7OA2 cassette, providing an internal control for pollen grain autofluorescence.

E) GFP:EXO70A2 localization in different stages of pollen tube elongation in the exo70a2 background.

F) GFP:EXO70A2 localization in a pollen tube in the WT background 
A

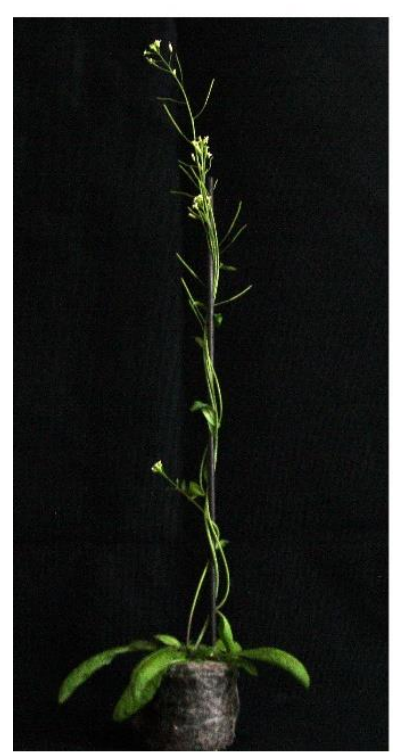

B exo70a1

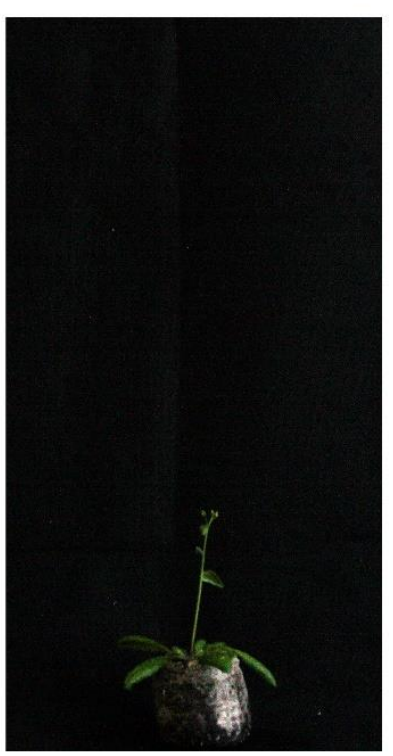

exo70a1 exo70a1 pA1::GFP:A1 pA1::GFP:A2

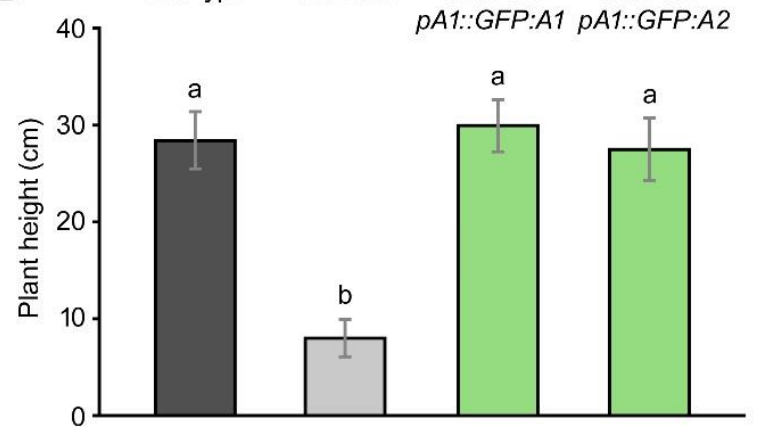

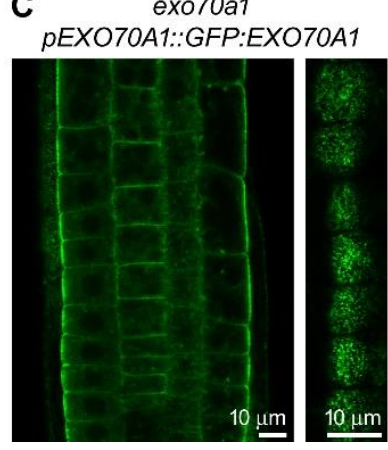

exo70a1

pEXO70A1::GFP:EXO70A1

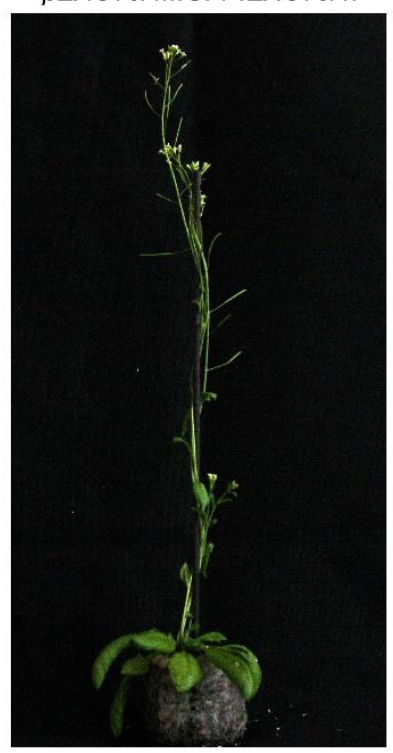

C exo70a1

PEXO70A1::GFP:EXO70A2

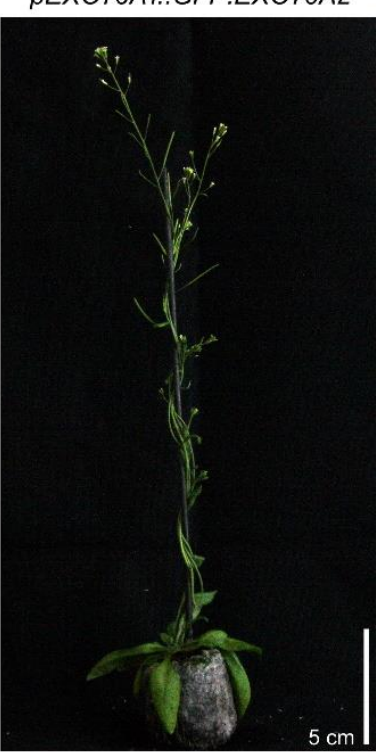

exo70a1

pEXO70A1::GFP:EXO70A2

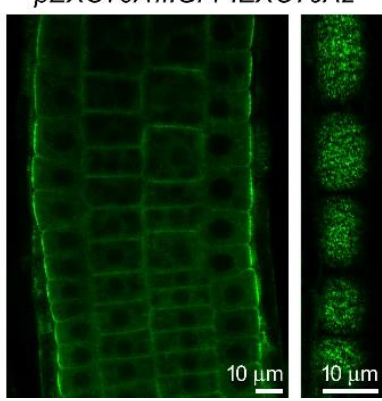

Figure 8. Ectopic expression of EXO70A2 in sporophytic tissues of exo70a1 mutant plants.

A) Representative images of 40-day-old plants document that the pEXO70A1::GFP:EXO70A2 expression in exo70a1 mutant plants rescued its growth defects similarly to pEXO70A1::GFP:EXO70A1.

B) Measurement of 40-day-old plant height. Bars represent SD; letters denote statistically different groups calculated by ANOVA at 0.01 significance level.

C) pEXO70A1::GFP:EXO70A2 shows very similar localization pattern to $p E X 070 A 1:: G F P: E X O 70 A 1$ in root epidermal cells. Left panels - confocal sections through the root transition zone. Right panels - confocal sections in the level of lateral plasma membranes. 


\section{SUPPLEMENTAL DATA}

File S1. EXO70 sequences used for the phylogenetic analysis.

Table S1. List of primers used in this study.

Figure S1. Characterization of the exo70a2 mutant line generated using the CRISPR/Cas9 system.

Figure S2. Development of WT and exo70a2 pollen.

Figure S3. Pollen germination efficiency is normal in complemented exo70a2 mutant plants.

\section{ACKNOWLEDGEMENTS}

This work was supported by the Czech Science Foundation (GAČR) - project 18-18290J, and part of the V.Z. income was covered by the Ministry of Education, Youth and Sports of the Czech Republic from European Regional Development Fund-Project "Centre for Experimental Plant Biology" CZ.02.1.01/0.0/0.0/16_019/0000738.

Microscopy was performed in the Laboratory of Confocal and Fluorescence Microscopy and the Microscopic facility of IEB. These facilities were supported by the European Regional Development FundProject and the state budget of the Czech Republic (projects no. CZ.1.05/4.1.00/16.0347 and CZ.2.16/3.1.00/21515), the Operational Programe Prague - Competitiveness (project no. CZ.2.16/3.1.00/21519), and the Czech-Biolmaging large RI project LM2015062.

We thank to Marta Čadyová for technical support and Juraj Sekereš for critical reading of the manuscript.

\section{REFERENCES}

Alexander MP (1969) Differential staining of aborted and nonaborted pollen. Stain Technol 44: 117-122 Backues SK, Korasick DA, Heese A, Bednarek SY (2010) The Arabidopsis dynamin-related protein2 family is essential for gametophyte development. Plant Cell 22: 3218-3231

Barrett T, Wilhite SE, Ledoux P, Evangelista C, Kim IF, Tomashevsky M, Marshall KA, Phillippy KH, Sherman PM, Holko M, et al (2013) NCBI GEO: archive for functional genomics data sets--update. Nucleic Acids Res 41: D991-5

Batystová K, Klejchová M, Janková-Drdová E, Synek L, Sabol P, Potocký M, Žárský V, Hála M (submitted) SEC15a and SEC15b isoforms of an exocyst subunit acquired specific roles in different Arabidopsis cell types.

Becker JD, Feijó JA (2007) How many genes are needed to make a pollen tube? Lessons from transcriptomics. Ann Bot 100: 1117-1123

Bloch D, Pleskot R, Pejchar P, Potocký M, Trpkošová P, Cwiklik L, Vukašinović N, Sternberg H, Yalovsky S, Žárský V (2016) Exocyst SEC3 and Phosphoinositides Define Sites of Exocytosis in Pollen Tube Initiation and Growth. Plant Physiol 172: 980-1002

Boyd C, Hughes T, Pypaert M, Novick P (2004) Vesicles carry most exocyst subunits to exocytic sites marked by the remaining two subunits, Sec3p and Exo70p. J Cell Biol 167: 889-901

Cai G, Parrotta L, Cresti M (2015) Organelle trafficking, the cytoskeleton, and pollen tube growth. J Integr Plant Biol 57: 63-78 
Chebli Y, Kaneda M, Zerzour R, Geitmann A (2012) The cell wall of the Arabidopsis pollen tube--spatial distribution, recycling, and network formation of polysaccharides. Plant Physiol 160: 1940-1955

Chebli Y, Kroeger J, Geitmann A (2013) Transport logistics in pollen tubes. Mol Plant 6: 1037-1052

Clough SJ, Bent AF (1998) Floral dip: a simplified method for Agrobacterium-mediated transformation of Arabidopsis thaliana. Plant J 16: 735-743

Cole RA, Synek L, Zarsky V, Fowler JE (2005) SEC8, a subunit of the putative Arabidopsis exocyst complex, facilitates pollen germination and competitive pollen tube growth. Plant Physiol 138: 2005-2018

Conze LL, Berlin S, Le Bail A, Kost B (2017) Transcriptome profiling of tobacco (Nicotiana tabacum) pollen and pollen tubes. BMC Genomics 18: 581

Curčková F, Eliáš M, Hála M, Obermeyer G, Žárský V (2001) Small GTPases and conserved signalling pathways in plant cell morphogenesis: From exocytosis to Exocyst. In Cell Biology of Plant and Fungal Tip Growth (Geitmann, A and Cresti, M, eds), Amsterdam: IOS Press, 105-122

Cvrčková F, Grunt M, Bezvoda R, Hála M, Kulich I, Rawat A, Zárský V (2012) Evolution of the land plant exocyst complexes. Front Plant Sci 3: 159

Davidson RM, Gowda M, Moghe G, Lin H, Vaillancourt B, Shiu S-H, Jiang N, Robin Buell C (2012) Comparative transcriptomics of three Poaceae species reveals patterns of gene expression evolution. Plant J 71: 492-502

De Smet R, Sabaghian E, Li Z, Saeys Y, Van de Peer Y (2017) Coordinated Functional Divergence of Genes after Genome Duplication in. Plant Cell 29: 2786-2800

Drdová EJ, Synek L, Pečenková T, Hála M, Kulich I, Fowler JE, Murphy AS, Zárský V (2013) The exocyst complex contributes to PIN auxin efflux carrier recycling and polar auxin transport in Arabidopsis. Plant J 73: 709-719

Elias M, Drdova E, Ziak D, BavInka B, Hala M, Curckova F, Soukupova H, Zarsky V (2003) The exocyst complex in plants. Cell Biol Int 27: 199-201

Fendrych M, Synek L, Pecenková T, Drdová EJ, Sekeres J, de Rycke R, Nowack MK, Zársky V (2013) Visualization of the exocyst complex dynamics at the plasma membrane of Arabidopsis thaliana. Mol Biol Cell 24: 510-520

Fendrych M, Synek L, Pecenková T, Toupalová H, Cole R, Drdová E, Nebesárová J, Sedinová M, Hála M, Fowler JE, et al (2010) The Arabidopsis exocyst complex is involved in cytokinesis and cell plate maturation. Plant Cell 22: 3053-3065

Goodstein DM, Shu S, Howson R, Neupane R, Hayes RD, Fazo J, Mitros T, Dirks W, Hellsten U, Putnam $\mathbf{N}$, et al (2012) Phytozome: a comparative platform for green plant genomics. Nucleic Acids Research 40: D1178-D1186

Grobei MA, Qeli E, Brunner E, Rehrauer H, Zhang R, Roschitzki B, Basler K, Ahrens CH, Grossniklaus U (2009) Deterministic protein inference for shotgun proteomics data provides new insights into Arabidopsis pollen development and function. Genome Res 19: 1786-1800

Guindon S, Dufayard J-F, Lefort V, Anisimova M, Hordijk W, Gascuel O (2010) New algorithms and methods to estimate maximum-likelihood phylogenies: assessing the performance of PhyML 3.0. Syst Biol 59: 307-321

Guo W, Grant A, Novick P (1999) Exo84p is an exocyst protein essential for secretion. J Biol Chem 274: 23558-23564

Hála M, Cole R, Synek L, Drdová E, Pecenková T, Nordheim A, Lamkemeyer T, Madlung J, Hochholdinger F, Fowler JE, et al (2008) An exocyst complex functions in plant cell growth in Arabidopsis and tobacco. Plant Cell 20: 1330-1345

He B, Xi F, Zhang X, Zhang J, Guo W (2007) Exo70 interacts with phospholipids and mediates the targeting of the exocyst to the plasma membrane. EMBO J 26: 4053-4065

Hepler PK, Winship $\sqcup$ (2015) The pollen tube clear zone: clues to the mechanism of polarized growth. J Integr Plant Biol 57: 79-92 
Hong D, Jeon BW, Kim SY, Hwang J-U, Lee Y (2016) The ROP2-RIC7 pathway negatively regulates lightinduced stomatal opening by inhibiting exocyst subunit Exo70B1 in Arabidopsis. New Phytol 209: 624-635

Hruz T, Laule O, Szabo G, Wessendorp F, Bleuler S, Oertle L, Widmayer P, Gruissem W, Zimmermann P (2008) Genevestigator v3: a reference expression database for the meta-analysis of transcriptomes. Adv Bioinformatics 2008: 420747

Jiang L, Yang S-L, Xie L-F, Puah CS, Zhang X-Q, Yang W-C, Sundaresan V, Ye D (2005) VANGUARD1 encodes a pectin methylesterase that enhances pollen tube growth in the Arabidopsis style and transmitting tract. Plant Cell 17: 584-596

Johnson MA, Preuss D (2002) Plotting a course: multiple signals guide pollen tubes to their targets. Dev Cell 2: 273-281

Kang B-H, Rancour DM, Bednarek SY (2003) The dynamin-like protein ADL1C is essential for plasma membrane maintenance during pollen maturation. Plant J 35: 1-15

Karimi M, Bleys A, Vanderhaeghen R, Hilson P (2007) Building Blocks for Plant Gene Assembly. Plant Physiology 145: 1183-1191

Katoh K, Standley DM (2013) MAFFT multiple sequence alignment software version 7: improvements in performance and usability. Mol Biol Evol 30: 772-780

Kaya H, Iwano M, Takeda S, Kanaoka MM, Kimura S, Abe M, Kuchitsu K (2015) Apoplastic ROS production upon pollination by RbohH and RbohJ in Arabidopsis. Plant Signal Behav 10: e989050

Kubátová Z, Pejchar P, Potocký M, Sekereš J, Žárský V, Kulich I (2019) Arabidopsis Trichome Contains Two Plasma Membrane Domains with Different Lipid Compositions Which Attract Distinct EXO70 Subunits. International Journal of Molecular Sciences 20: 3803

Kulich I, Cole R, Drdová E, Cvrcková F, Soukup A, Fowler J, Zárský V (2010) Arabidopsis exocyst subunits SEC8 and EXO7OA1 and exocyst interactor ROH1 are involved in the localized deposition of seed coat pectin. New Phytol 188: 615-625

Kulich I, Pečenková T, Sekereš J, Smetana O, Fendrych M, Foissner I, Höftberger M, Zárský V (2013) Arabidopsis exocyst subcomplex containing subunit EXO70B1 is involved in autophagy-related transport to the vacuole. Traffic 14: 1155-1165

Kulich I, Vojtíková Z, Glanc M, Ortmannová J, Rasmann S, Žárský V (2015) Cell wall maturation of Arabidopsis trichomes is dependent on exocyst subunit EXO7OH4 and involves callose deposition. Plant Physiol 168: 120-131

Lassig R, Gutermuth T, Bey TD, Konrad KR, Romeis T (2014) Pollen tube NAD(P)H oxidases act as a speed control to dampen growth rate oscillations during polarized cell growth. Plant J 78: 94-106

Leroux C, Bouton S, Kiefer-Meyer M-C, Fabrice TN, Mareck A, Guénin S, Fournet F, Ringli C, Pelloux J, Driouich A, et al (2015) PECTIN METHYLESTERASE48 is involved in Arabidopsis pollen grain germination. Plant Physiol 167: 367-380

Loraine AE, McCormick S, Estrada A, Patel K, Qin P (2013) RNA-seq of Arabidopsis pollen uncovers novel transcription and alternative splicing. Plant Physiol 162: 1092-1109

MacAlister CA, Ortiz-Ramírez C, Becker JD, Feijó JA, Lippman ZB (2016) Hydroxyproline Oarabinosyltransferase mutants oppositely alter tip growth in Arabidopsis thaliana and Physcomitrella patens. Plant J 85: 193-208

Matern HT, Yeaman C, Nelson WJ, Scheller RH (2001) The Sec6/8 complex in mammalian cells: characterization of mammalian Sec3, subunit interactions, and expression of subunits in polarized cells. Proc Natl Acad Sci U S A 98: 9648-9653

Mori T, Kuroiwa H, Higashiyama T, Kuroiwa T (2006) GENERATIVE CELL SPECIFIC 1 is essential for angiosperm fertilization. Nat Cell Biol 8: 64-71

Paul P, Röth S, Schleiff E (2016) Importance of organellar proteins, protein translocation and vesicle transport routes for pollen development and function. Plant Reprod 29: 53-65 
Pecenková T, Hála M, Kulich I, Kocourková D, Drdová E, Fendrych M, Toupalová H, Zársky V (2011) The role for the exocyst complex subunits Exo70B2 and Exo70H1 in the plant-pathogen interaction. J Exp Bot 62: 2107-2116

Pecenková T, Markovic V, Sabol P, Kulich I, Žárský V (2017) Exocyst and autophagy-related membrane trafficking in plants. J Exp Bot 69: 47-57

Peng J, Ilarslan H, Wurtele ES, Bassham DC (2011) AtRabD2b and AtRabD2c have overlapping functions in pollen development and pollen tube growth. BMC Plant Biol 11: 25

Pleskot R, Cwiklik L, Jungwirth P, Žárský V, Potocký M (2015) Membrane targeting of the yeast exocyst complex. Biochim Biophys Acta 1848: 1481-1489

Potocký M, Jones MA, Bezvoda R, Smirnoff N, Zárský V (2007) Reactive oxygen species produced by NADPH oxidase are involved in pollen tube growth. New Phytol 174: 742-751

Potocký M, Pejchar P, Gutkowska M, Jiménez-Quesada MJ, Potocká A, Alché J de D, Kost B, Žárský V (2012) NADPH oxidase activity in pollen tubes is affected by calcium ions, signaling phospholipids and Rac/Rop GTPases. J Plant Physiol 169: 1654-1663

Proost S, Mutwil M (2018) CoNekT: an open-source framework for comparative genomic and transcriptomic network analyses. Nucleic Acids Res 46: W133-W140

Qin Y, Dong J (2015) Focusing on the focus: what else beyond the master switches for polar cell growth? Mol Plant 8: 582-594

Rawat A, Brejšková L, Hála M, Curčková F, Žárský V (2017) The Physcomitrella patens exocyst subunit EXO70.3d has distinct roles in growth and development, and is essential for completion of the moss life cycle. New Phytol 216: 438-454

Ronquist F, Teslenko M, van der Mark P, Ayres DL, Darling A, Höhna S, Larget B, Liu L, Suchard MA, Huelsenbeck JP (2012) MrBayes 3.2: efficient Bayesian phylogenetic inference and model choice across a large model space. Syst Biol 61: 539-542

Rossetti S, Bonatti PM (2001) In situ histochemical monitoring of ozone- and TMV-induced reactive oxygen species in tobacco leaves. Plant Physiology and Biochemistry 39: 433-442

Rybak K, Steiner A, Synek L, Klaeger S, Kulich I, Facher E, Wanner G, Kuster B, Zarsky V, Persson S, et al (2014) Plant cytokinesis is orchestrated by the sequential action of the TRAPPII and exocyst tethering complexes. Dev Cell 29: 607-620

Sabol P, Kulich I, Žárský V (2017) RIN4 recruits the exocyst subunit EXO70B1 to the plasma membrane. J Exp Bot 68: 3253-3265

Sekereš J, Pejchar P, Šantrůček J, Vukašinović N, Žárský V, Potocký M (2017) Analysis of Exocyst Subunit EXO70 Family Reveals Distinct Membrane Polar Domains in Tobacco Pollen Tubes. Plant Physiol 173: 1659-1675

Smirnova AV, Matveyeva NP, Yermakov IP (2014) Reactive oxygen species are involved in regulation of pollen wall cytomechanics. Plant Biol 16: 252-257

Synek L, Schlager N, Eliás M, Quentin M, Hauser M-T, Zárský V (2006) AtEXO70A1, a member of a family of putative exocyst subunits specifically expanded in land plants, is important for polar growth and plant development. Plant J 48: 54-72

Synek L, Vukašinović N, Kulich I, Hála M, Aldorfová K, Fendrych M, Žárský V (2017) EXO70C2 Is a Key Regulatory Factor for Optimal Tip Growth of Pollen. Plant Physiol 174: 223-240

TerBush DR, Maurice T, Roth D, Novick P (1996) The Exocyst is a multiprotein complex required for exocytosis in Saccharomyces cerevisiae. EMBO J 15: 6483-6494

Tian G-W, Chen M-H, Zaltsman A, Citovsky V (2006) Pollen-specific pectin methylesterase involved in pollen tube growth. Dev Biol 294: 83-91

Vogler F, Schmalzl C, Englhart M, Bircheneder M, Sprunck S (2014) Brassinosteroids promote Arabidopsis pollen germination and growth. Plant Reprod 27: 153-167 
Vukašinović N, Curčková F, Eliáš M, Cole R, Fowler JE, Žárský V, Synek L (2014) Dissecting a hidden gene duplication: the Arabidopsis thaliana SEC10 locus. PLoS One 9: e94077

Vukašinović N, Oda Y, Pejchar P, Synek L, Pečenková T, Rawat A, Sekereš J, Potocký M, Žárský V (2017) Microtubule-dependent targeting of the exocyst complex is necessary for xylem development in Arabidopsis. New Phytol 213: 1052-1067

Wang Z-P, Xing H-L, Dong L, Zhang H-Y, Han C-Y, Wang X-C, Chen Q-J (2015) Egg cell-specific promotercontrolled CRISPR/Cas9 efficiently generates homozygous mutants for multiple target genes in Arabidopsis in a single generation. Genome Biol 16: 144

Waterhouse AM, Procter JB, Martin DMA, Clamp M, Barton GJ (2009) Jalview Version 2--a multiple sequence alignment editor and analysis workbench. Bioinformatics 25: 1189-1191

Žárský V, Kulich I, Fendrych M, Pečenková T (2013) Exocyst complexes multiple functions in plant cells secretory pathways. Current Opinion in Plant Biology 16: 726-733

Žárský V, Sekereš J, Kubátová Z, Pečenková T, Curčková F (2019) Three subfamilies of exocyst EXO70 family subunits in land plants: early divergence and ongoing functional specialization. Journal of Experimental Botany. doi: 10.1093/jxb/erz423

Zhang X, Pumplin N, Ivanov S, Harrison MJ (2015) EXO70I Is Required for Development of a Sub-domain of the Periarbuscular Membrane during Arbuscular Mycorrhizal Symbiosis. Curr Biol 25: 2189-2195

Zhao L-J, Yuan H-M, Guo W-D, Yang C-P (2016) Digital Gene Expression Analysis of Populus simonii $\times$ P. nigra Pollen Germination and Tube Growth. Frontiers in Plant Science. doi: 10.3389/fpls.2016.00825 